\title{
PRIMARY EPITHELIAL TUMOURS OF THE JAW
}

BY

\author{
T. F. HEWER \\ From the Department of Pathology, University of Bristol
}

(RECEIVED FOR PUBLICATION MARCH 3, 1952)

Primary epithelial tumours in the jaw are an interesting group in that they may be derived from the lining epithelium of the mouth or from that which has invaded the jaw during the embryonic development of the teeth. The exact origin of these tumours is often debatable, their diagnosis radiologically and histologically not always easy, and their prognosis therefore in doubt.

The morphological possibilities of epithelial tumours of the jaw can only be appreciated if the details of the embryology of the teeth are borne in mind. It will be remembered that the ectodermal lining of the mouth grows into the primitive gum in the form of a solid dental lamina in the seventh week of development. At intervals along this lamina thickenings of the epithelium appear and constitute the enamel organs. At a later stage, as in the 16 weeks' emb'yo illustrated in Fig .1, the enamel organ comes to lie over the mesenchymal dental papilla and has a central core of so-called stellate reticulum, which is a further differentiation of the epithelium, and a marginal layer of columnar enamel-forming ameloblasts. Ordinarily the stellate reticulum, the outer layer of ameloblasts, and the dental lamina disappear by the time the deciduous and permanent teeth are formed, but remnants of this epithelium may occasionally remain and give rise to neoplastic growth at a later date.

It is important to remember that the adult oral mucosa, composed of squamous epithelium, of ectodermal or entodermal origin, has a capacity at least as great as that of the epithelium of the body surface to produce the whole range of epithelial tumours: simple inclusion and retention cysts, squamous cell carcinoma, basal cell tumours of " rodent" type, and adamantinomas. Any of these may invade the jaw and on occasion it is difficult to decide whether the tumour arose from an intraalveolar site or from the mucous surface.

Most tumours in the jaw declare their presence by causing a swelling, and on radiological examination an area of absorption of bone is generally detected. It is not the purpose of this paper to discuss the diagnostic radiological features. Our experience in the Bristol Bone Tumour Register has been that one cannot always rely upon radiological appearances, and unfortunately a histological examination of a biopsy specimen may be misleading. Taken together the two methods generally enable one to make an accurate diagnosis, but only if the limitations of both are borne in mind.

In a study over a period of six years of 10 examples of simple dental cysts, 12 adamantinomas, and five intra-alveolar carcinomas, and various carcinomas of the mouth cavity invading the jaw, we have been impressed particularly by the frequency with which a wrong clinical diagnosis has been made and by the hazards of histological diagnosis on small biopsy specimens. A few examples of these different types of epithelial tumour, some benign and some malignant, will serve to illustrate these features.

\section{Benign Epithelial Cysts}

Simple periodontal cysts may be derived from epithelial cell nests (of Mallassez) in the area of the periodontal membrane, or from the remnants of the deep part of the developmental dental lamina. They are lined by a very simple squamous epithelium which is often damaged by secondary infection of the fluid or keratinous contents. Toller (1948) has shown that the hydrostatic pressure within these cysts may be as much as $70 \mathrm{~cm}$. of water, so it is not surprising that they cause pressure atrophy of the bone in which they are embedded and occasionally become quite large.

A periodontal cyst may also result if the original infection in a periapical granuloma clears up and leaves a cavity which becomes lined by squamous epithelium and contains epithelial debris and cholesterol crystals. There is generally a dense fibrous wall with evidence of chronic inflammation.

Another simple tumour occurring in the jaw is a dentigerous cyst, of which Fig. 2 is an example. This arises alongside a deciduous tooth and encloses the permanent tooth below it : it is lined by squamous epithelium and can be recognized only by its characteristic relationship to the teeth. 


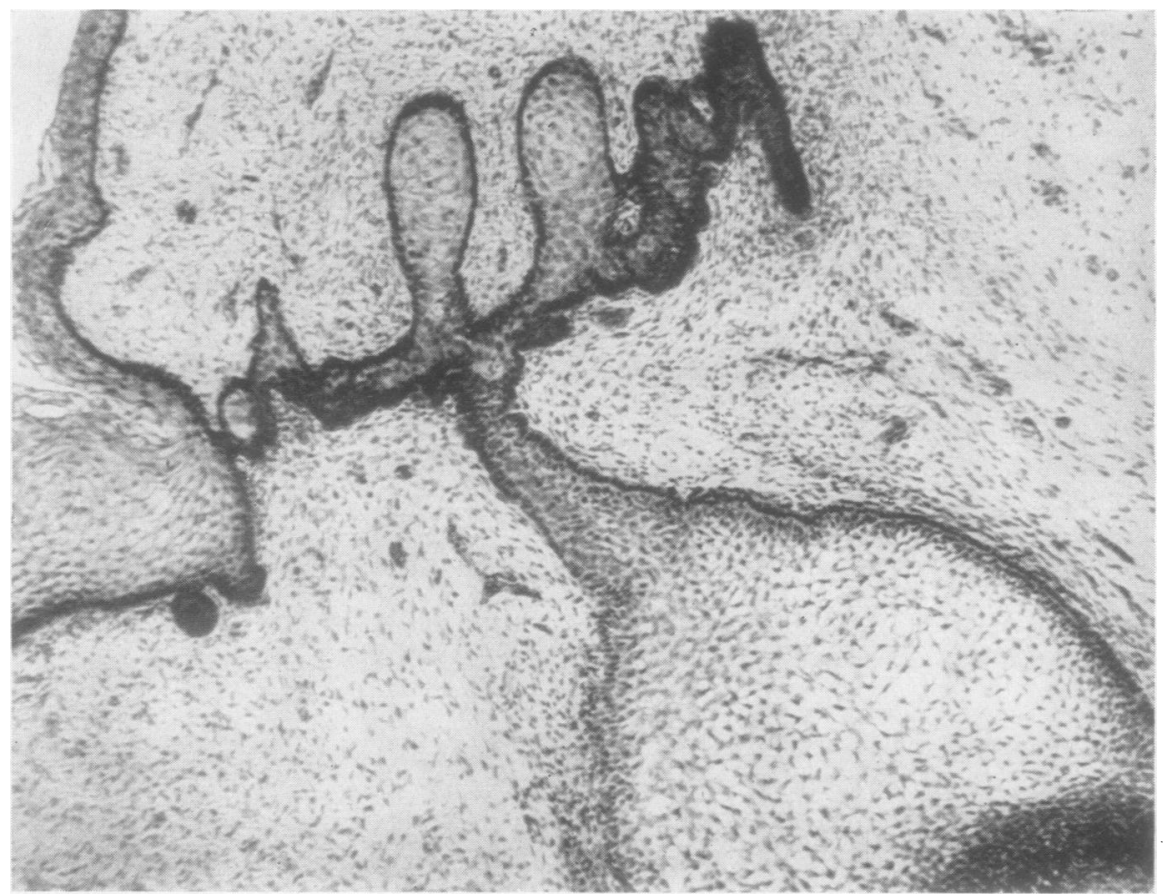

Fig. 1.-Deciduous tooth germ of 16 weeks embryo. The oral mucosa is on the left. The dental lamina arising from it contains two masses of squamous epithelium outlined by cubical basal cells. The enamel organ, descending from the lamina, has a central core of stellate epithelial "reticulum" with an outer limiting layer of columnar ameloblasts. The concave base of the enamel organ is a cellular mass of larger ameloblasts lying on the mesenchyme of the tooth germ, $\times 150$.

All of these benign cysts are unilocular and have a clear-cut outline radiographically. Compound odontomes are congenital malformations, or else result from inflammation of the tooth germ, and we need not consider them here.

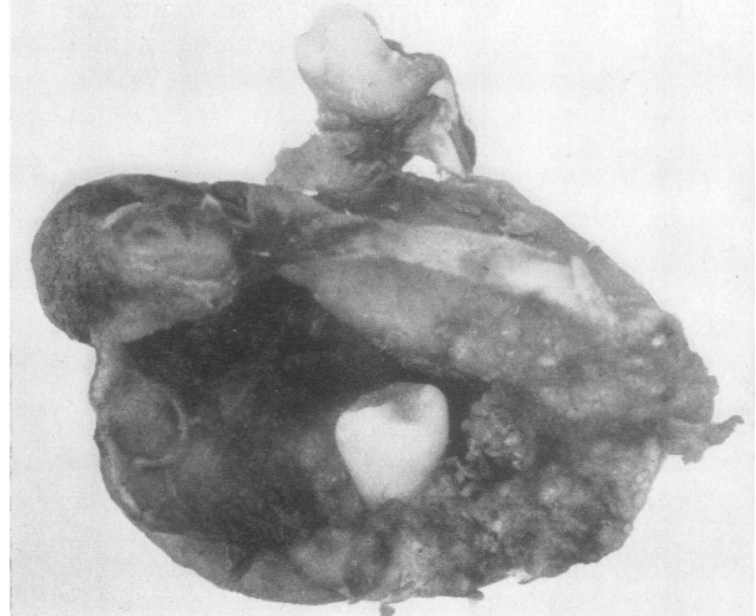

FiG. 2.-The dental cyst, arising in association with the root of the deciduous tooth, encloses the crown of the permanent tooth below it.

\section{Malignant Epithelial Tumours}

It is with the invasive or malignant tumours that the chief histological difficulty is encountered because the biopsy specimen may have a deceptively simple appearance. The commonest tumour in this group is the adamantinoma which is generally supposed to arise from the outer layer of ameloblasts of the enamel organ and to be locally invasive. It is characterized histologically by rounded or elongated islands of epithelium with a well-defined margin of columnar cells, similar to those of the enamel organ, and a central area of stellate reticulum which is also epithelial and may contain large or small cystic spaces. They are commonly multilocular and have a "soap-bubble" appearance radiographically, but unfortunately this is not an entirely reliable criterion for diagnosis.

It must be remembered that histologically similar tumours may be found elsewhere in the oral, nasal, and pharyngeal cavities; in the region of the pituitary as components of a craniopharyngioma; and in the tibia and other bones. And areas resembling an adamantinoma are occasionally seen in a squamous carcinoma of the surface skin, so one cannot limit their origin to the enamel organ. 
A few details of seven selected cases of malignant epithelial tumours of the jaw will serve to illustrate some of the diagnostic pitfalls.

\section{Case Reports}

Case 1 (B.T.R. 129). - In 1937 a woman aged 35 had an operation for evacuation of a " dentigerous cyst " of the mandible. Next year there was a local recurrence and the condition was described as an "odontoma": the cavity was scraped out. In 1942 she was thought to have fibrocystic disease of the jaw and a cyst was removed and the bony walls of the cavity crushed inwards. In 1945 the cyst recurred and a fourth conservative operation was performed.

On November 24, 1947, the left ramus was found expanded and a radiograph showed the characteristic "soap-bubble" appearance of an adamantinoma. The left half of the mandible was completely removed and the patient has remained free of recurrence.

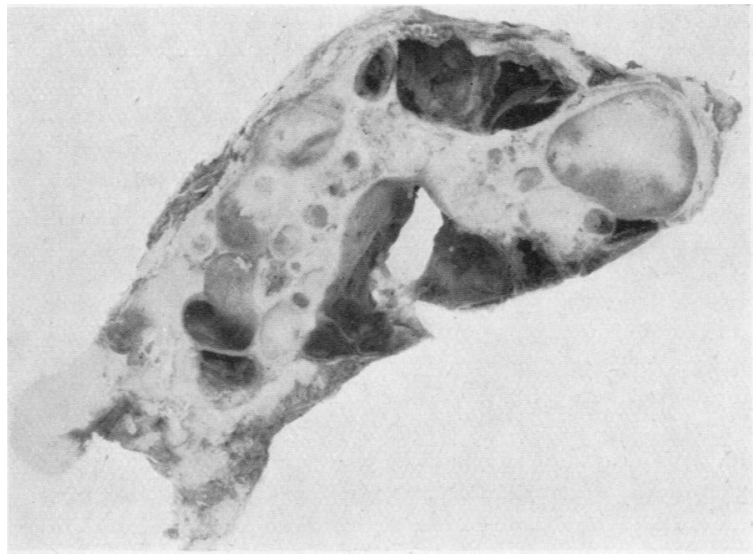

Fig. 3.-Mandibular ramus completely filled with adamantinomatous cysts.

The specimen (Fig. 3) consisted of an expanded mandibular ramus with numerous, smooth-lined cysts containing glairy fluid or, in some cases, haemorrhagic contents. Histologically (Fig. 4) the cystic spaces were due to degeneration of the stellate reticulum which, in places, was undergoing squamous metaplasia.

At this stage there was no possibility of mistaking the $x$-ray or the histological appearance.

Case 2 (B.T.R. 180). - In 1922 a woman aged 22 developed a swelling in the left side of the mandible. A number of small local operations were performed during the next 20 years with no histological examination. In 1942 a more radical local removal was done, but by 1949 the swelling recurred once more and the $x$-ray appearance was characteristic of adamantinoma absorbing a large part of the jaw. In March, 1949, the whole of the left half of the mandible was removed. In August, 1951, she was very well and declined a bone graft because with a lower denture she could manage perfectly and showed very little deformity.

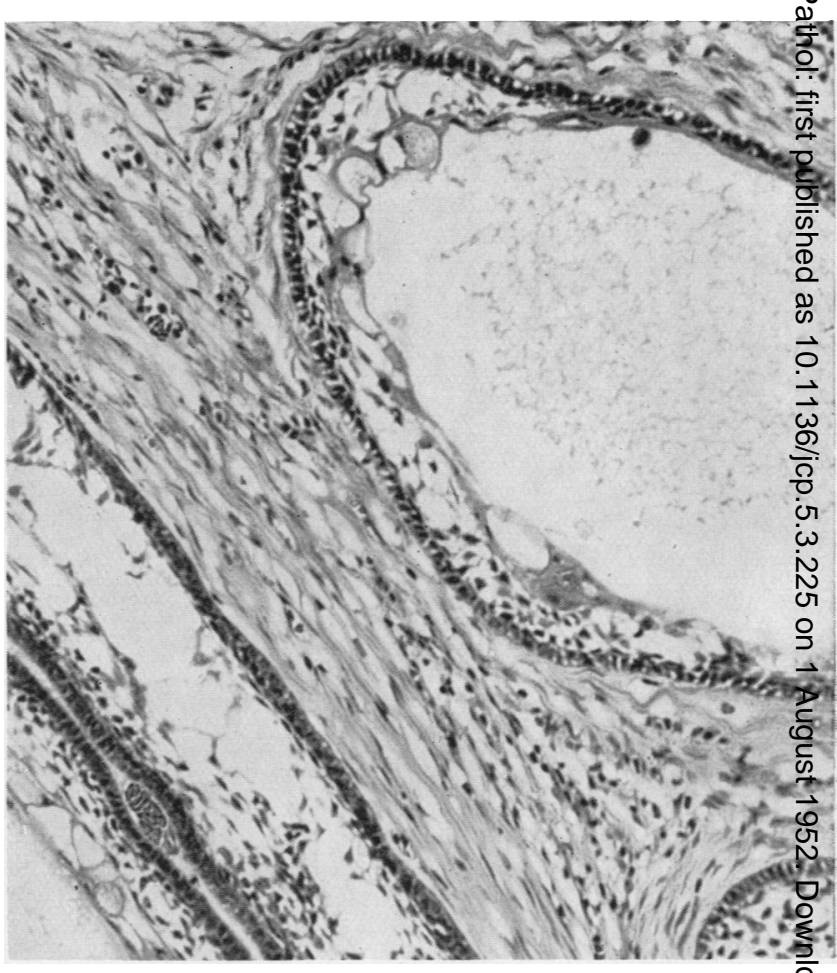

FIG. 4.-Section from the edge of one of the cysts in Fig. 3 showing characteristic degeneration of the stellate reticulum and marginal layers of colummar ameloblasts. The stellate cells lining the small cyst in the centre Ge showing some squamous differentiation, $\times 200$.

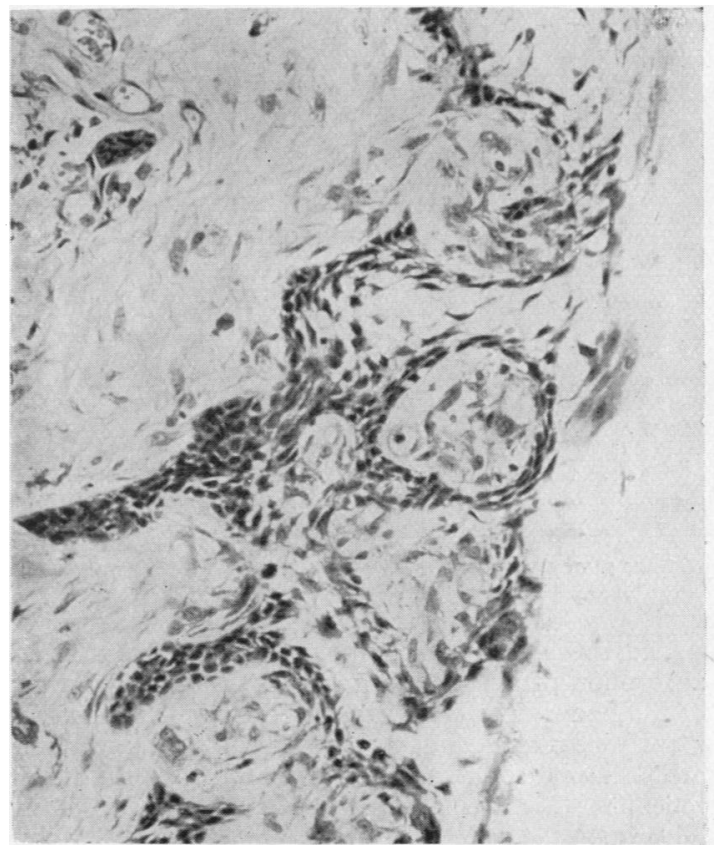

Fig. 5.-Degenerate lining of a large adamantinomatous cyst. Cubical basal cells are covering vascular fibrous projections and some ragged stellate epithelium is recognizable, $\times 200$. 
The specimen contained several small and two large cysts. The lining of the larger cysts (Fig. 5) was degenerate but still recognizable as adamantinoma. Papillary fibrous and vascular projections were covered by epithelium of rather basal-cell type, with fragments of stellate reticulum. Haemorrhage into the cysts had occurred from the vascular stroma. The small cysts were almost solid masses of adamantinoma with minute cysts resulting from the degeneration of the stellate reticulum. Fig. 6 shows also some squamous metaplasia of this reticulum.

Case 3 (B.T.R. 120). - In 1937 a woman aged 46 had a "dental cyst" removed from the left side of the mandible. It recurred in 1939, 1945, and 1947 after further local removals, no histological examination being made. In March, 1948, at the age of 58, most of the left half of the mandible was removed with success.

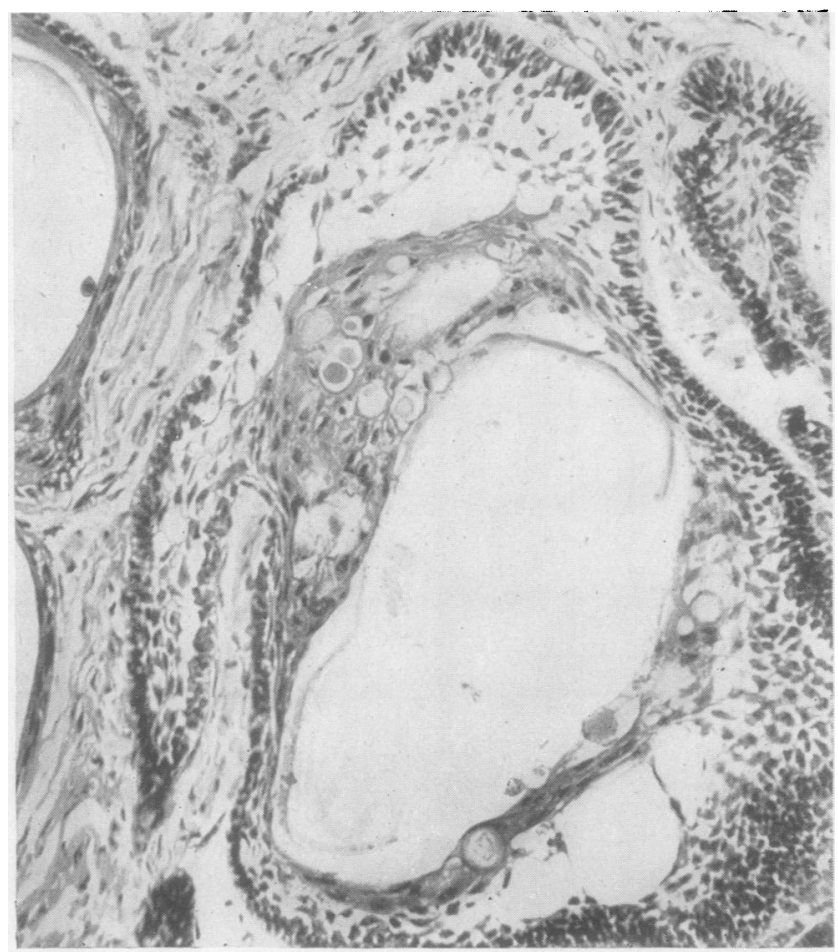

Fig. 6.-From the same section as Fig. 5. A clearer picture of an adamantinoma, with some squamous metaplasia of small cysts, $\times 200$.

The specimen (Fig. 7) showed cystic dilatation and absorption of almost the whole of the ramus and part of the body. The cysts were lined by typical adamantinomatous tissue and the more exuberantly granular areas showed some squamous metaplasia of the stellate reticulum (Fig. 8). In the lower central part of this illustration the squamous epithelium looks like a poorly differentiated squamous carcinoma, and if a small

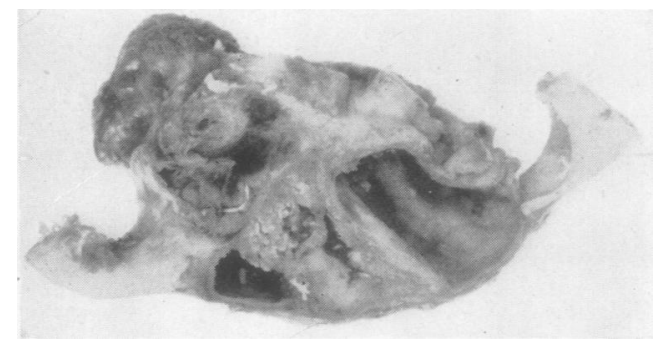

FIG. 7.-Ramus and large part of body of mandible occupied by adamantinomatous cysts.

biopsy had been taken it is possible that such a diagnosis might have been made. In fact the tumour behaved like an adamantinoma, invaded locally, and did not spread to lymph nodes.

Case 4 (B.T.R. 168). - In December, 1948, a man aged 58 was seen with a painless swelling of the right lower jaw of seven months' duration. A radiograph showed several cysts invading the jaw and a diagnosis of adamantinoma was made. The right half of the mandible was removed and replaced by a chondro-osseous graft from a rib. In August, 1951, there was no recurrence.

The specimen contained some five cysts, about $1 \mathrm{~cm}$. in diameter, one extending into the condyle and another into the masseter muscle. They were lined with smooth, pearly-looking epithelium and contained friable keratinous material. Histologically (Fig. 9) there was a uniform lining of squamous epithelium with an orderly rather columnar layer at the base and no suggestion of alveolar arrangement, stellate reticulum, or any other evidence of adamantinoma; and yet it was behaving like an adamantinoma and had that gross and radiographic appearance. It seemed that this tumour must have been present for a very long time and produced no symptoms until it invaded the masseter muscle. The final histological appearance may be interpreted as a complete squamous metaplasia of an adamantinoma, although there is of course no proof that it ever had the characteristic adamantinomatous pattern. The important point is that if complete reliance had been placed upon its histological appearance the invasive nature of the growth would not have been suspected.

Case 5 (B.T.R. 323).-A woman aged 71 had had an ulcer for two years in an edentulous area of the right maxillary alveolus posteriorly. Radiologically there was absorption of the bony floor of the antrum, and a biopsy (Fig. 10) showed a basal cell carcinoma arising from the buccal mucosa. In April, 1951, the whole of the right maxilla was avulsed from the pterygoid plates and a stent mould covered with Thiersch graft was packed into the cavity. Further histological examination of the tumour showed a remarkable and gradual transition from a basal cell carcinoma to an alveolar epithelial tumour and, in the deeper part, to a perfectly characteristic adamantinoma (Fig. 11). 
It is our opinion that this ulcer, which was never more than $1 \mathrm{~cm}$. in diameter, was a basal cell carcinoma arising in the buccal mucosa and assumed the histological guise of an adamantinoma as it invaded the maxilla.

Case 6 (B.T.R. 399).-A woman aged 22 had a history of a progressive painful swelling of the right side of the mandible for eight months before she was seen and found, on $x$-ray examination, to have a cyst surrounding an unerupted third molar tooth. This was apparently a dentigerous cyst, but because of its rapid development and considerable extent, a biopsy was made before surgical treatment. This showed a characteristic adamantinomatous histological pattern, and in spite of the unilocular appearance it was treated as an adamantinoma, half the mandible being resected. On gross examination it was a dentigerous cyst, but in places the epithelial lining was coarsely granular and thicker than usual (Fig. 12). The less granular parts were lined by simple squamous epithelium with no invasion of the underlying bone, but in the thicker areas there were long pegs of epithelium invading deeply and becoming transformed into an adamantinoma (Fig. 13) which was invading the bone beyond the limits of the cyst. This case was interpreted as an example of a dentigerous cyst in which

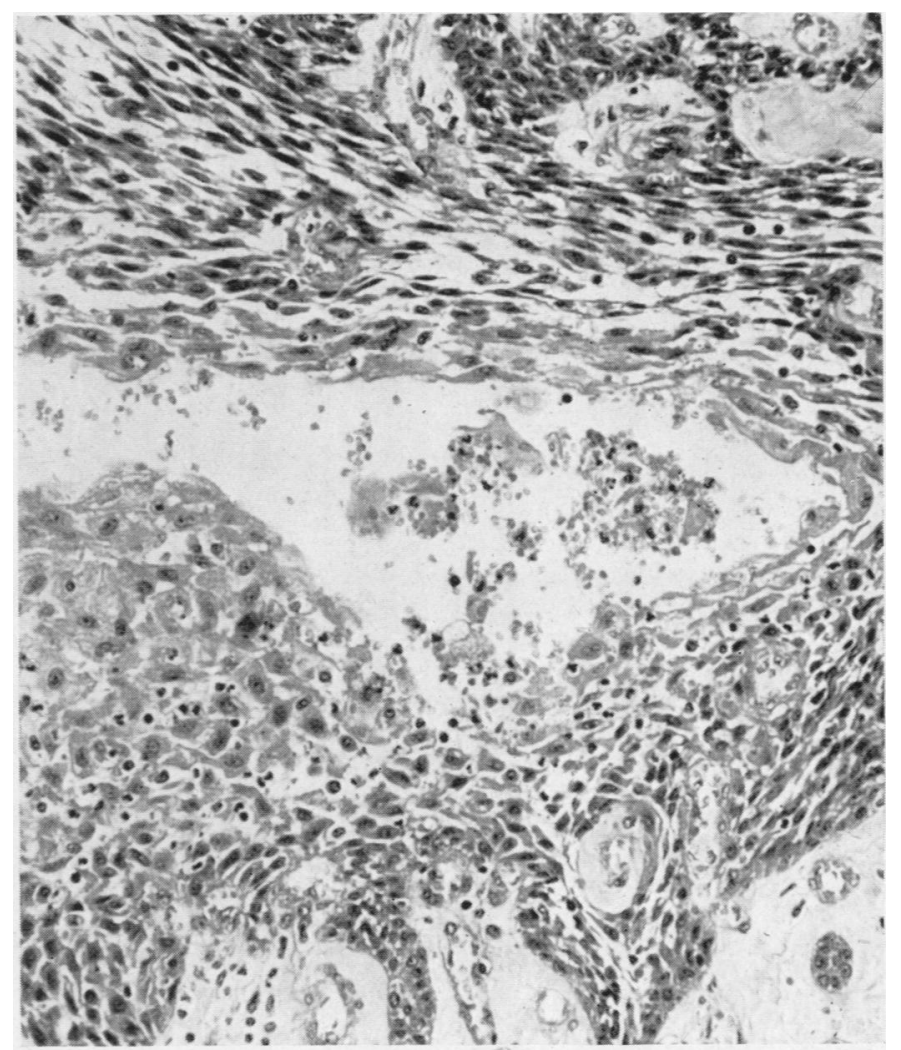

FIG. 8.-From the lining of one of the large cysts in Fig. 7. Note the considerable squamous metaplasia towards the lower right corner, resembling a squamous cell carcinoma. The rest of the picture is a pure adamantinoma. $\times 200$.

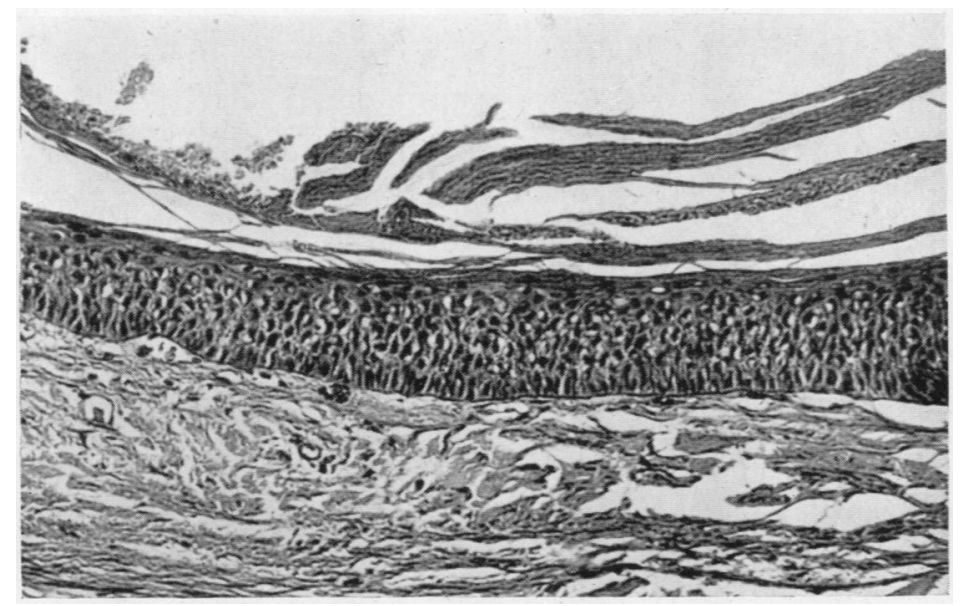

Fig. 9.-Wall of an adamantinomatous cyst of mandible with a uniform squamous epithelial lining, no stellate reticulum but inspissated keratinous contents. There is a columnar cell basal layer. $\times 200$. 
the squamous epithelial lining suffered an adamantinomatous change. If a biopsy had not been taken the cyst might well have been removed conservatively and the adamantinomatous invasion of the adjacent bone left behind.

Case 7 (B.T.R. 170).--In July, 1948, a woman aged 75 had her right lower third molar extracted because of severe toothache. The pain continued and she was treated with penicillin for osteomyelitis. A radiograph showed local bony rarefaction. By November, 1948, a swelling was visible in the mouth, but the mucosa was intact. A radiograph then showed extreme absorption of the bone of the ascending ramus and part of the body of the mandible approaching spontaneous fracture. A biopsy was performed and diagnosed angiosarcoma. In December, 1948, the whole of the right half of the mandible and the masseter muscle were removed; one of the upper cervical lymph nodes contained tumour, as did some at the angle of the jaw. Shortly after the operation the tumour recurred, did not respond to radiotherapy, and caused the death of the patient. The tumour (Fig. 14) was solid and pale grey, had destroyed a large part of the mandible, and diffusely invaded the

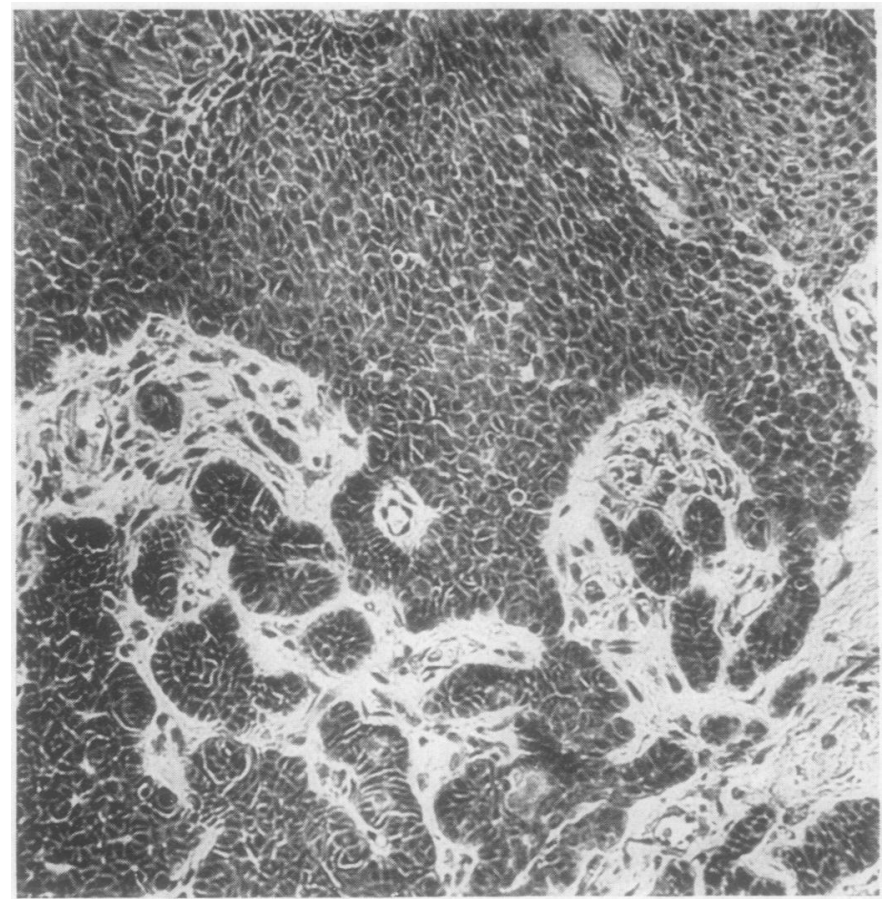

Fig. 10.-Basal cell carcinoma arising from oral mucosa on alveolus. Original biopsy. $\times 200$.

adjacent muscles. There was ulceration of the overlying mucosa, but this had developed terminally. Histologically the tumour (Fig. 15) was a poorly differentiated, Grade 3 or 4, squamous carcinoma with a tendency to form alveoli. In some areas (Fig. 16) the alveolar pattern was dominant, but nowhere were there any columnar cells or any trace of stellate reticulum. It was not possible therefore to adduce an origin from the enamel organ, but all the clinical evidence was that it arose within the mandible, involving the overlying mucosa quite late, and this, together with the curious alveolar histological appearance, led us to call it an intra-alveolar squamous cell carcinoma.

\section{Discussion and Conclusions}

Cases 1, 2, and 3 are representative of many in which it was assumed originally that the lesion was a simple dental cyst and in consequence inadequate local operations were performed. If the true nature of these neoplasms had been appreciated early it would undoubtedly have been possible to operate adequately and avoid the necessity of ultimately removing half the mandible. So many of these cases have
Fig. 11.-A deeper section after operation from the same case as in Fig. 10. The cystic spaces in this field are in the connective tissue stroma, not
in the stellate reticulum which is almost solid. $\times 200$. 
Fig. 12.-Dentigerous cyst with adamantinoma arising in its wall and invading mandible.

Fig. 13.-Lining of cyst in Fig. 12 showing superficial squamous epithelium with adamantinomatous pegs passing deeply into the jaw. $\times 200$.
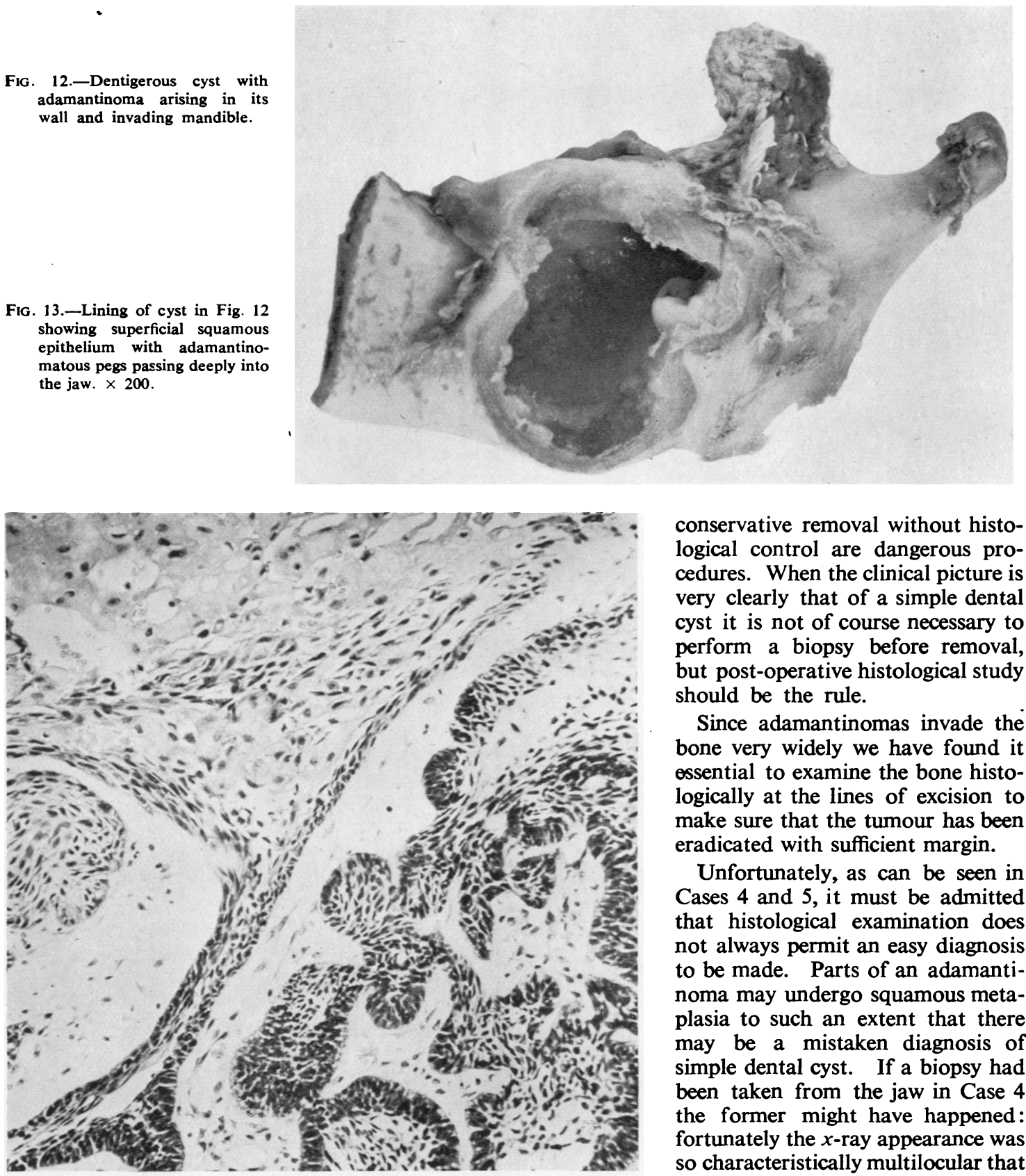

conservative removal without histological control are dangerous procedures. When the clinical picture is very clearly that of a simple dental cyst it is not of course necessary to perform a biopsy before removal, but post-operative histological study should be the rule.

Since adamantinomas invade the bone very widely we have found it essential to examine the bone histologically at the lines of excision to make sure that the tumour has been eradicated with sufficient margin.

Unfortunately, as can be seen in Cases 4 and 5 , it must be admitted that histological examination does not always permit an easy diagnosis to be made. Parts of an adamantinoma may undergo squamous metaplasia to such an extent that there may be a mistaken diagnosis of simple dental cyst. If a biopsy had been taken from the jaw in Case 4 the former might have happened: fortunately the $x$-ray appearance was so characteristically multilocular that radical operation was performed

come our way with a similar history of inadequate operations that we believe a histological study should be made of every dental cyst that is diagnosed, whether or not it is radiologically unilocular, and even if it is quite clearly a dentigerous cyst. Local crushing operations and without any hesitation. On the other hand, if the squamous metaplasia is of an undifferentiated type it may be very difficult to distinguish the tumour from an intra-alveolar epidermoid carcinoma. Willis (1948) refers to this difficulty and prefers to call the whole group "carcinomas of the tooth-germ 


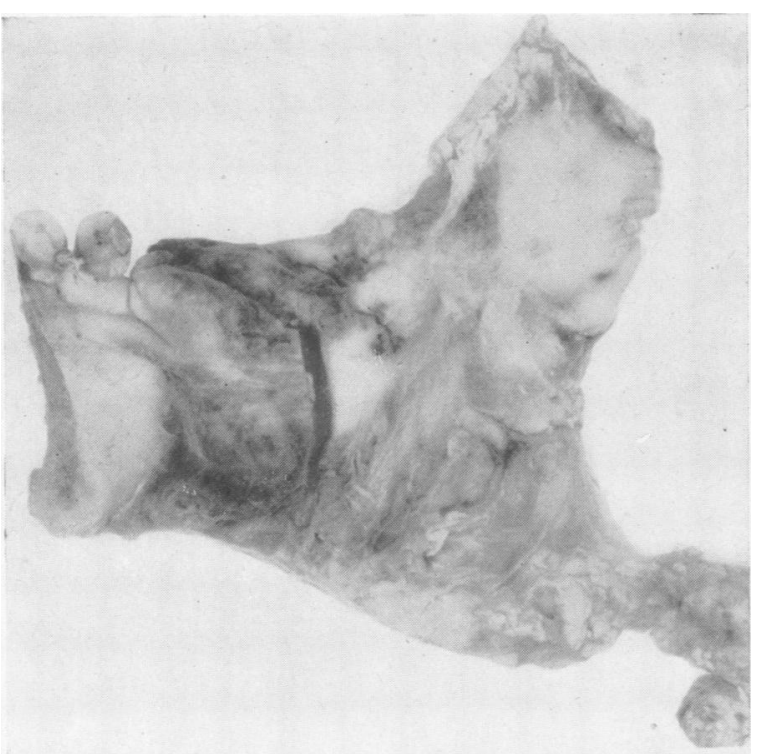

FIG. 14. - Intra-alveolar carcinoma ulcerating into mouth and invading masseter muscle and local lymph nodes.

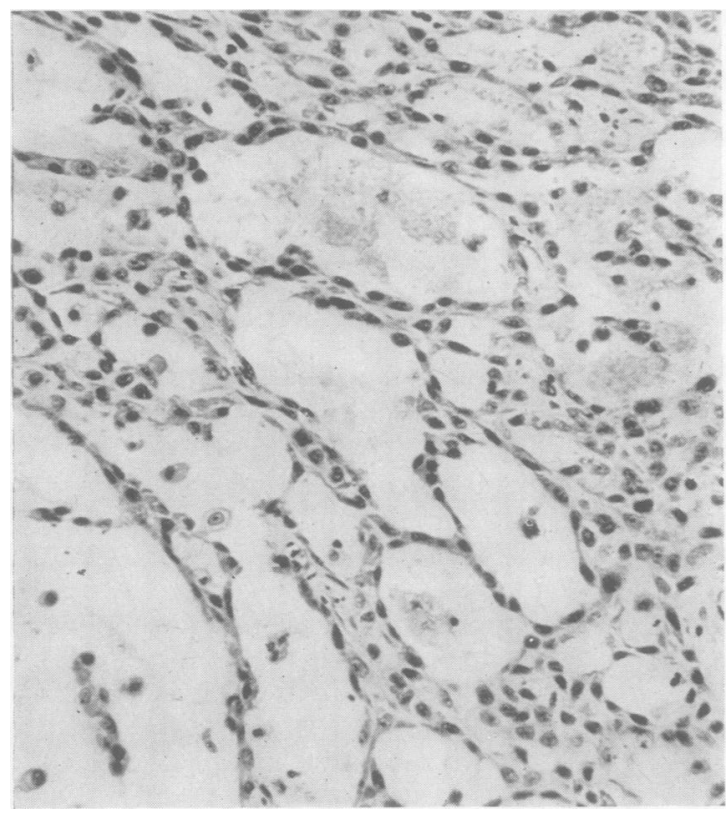

Fig. 16.-Intra-alveolar portion of same tumour as Fig. 15 showing alveolar pattern. $\times 200$.

residues," but this diagnosis, without amplification, would be insufficient for the surgeon who wants to know whether the tumour is likely to remain within the jaw and grow slowly or invade the soft tissues and metastasize by lymph or blood stream. The intra-

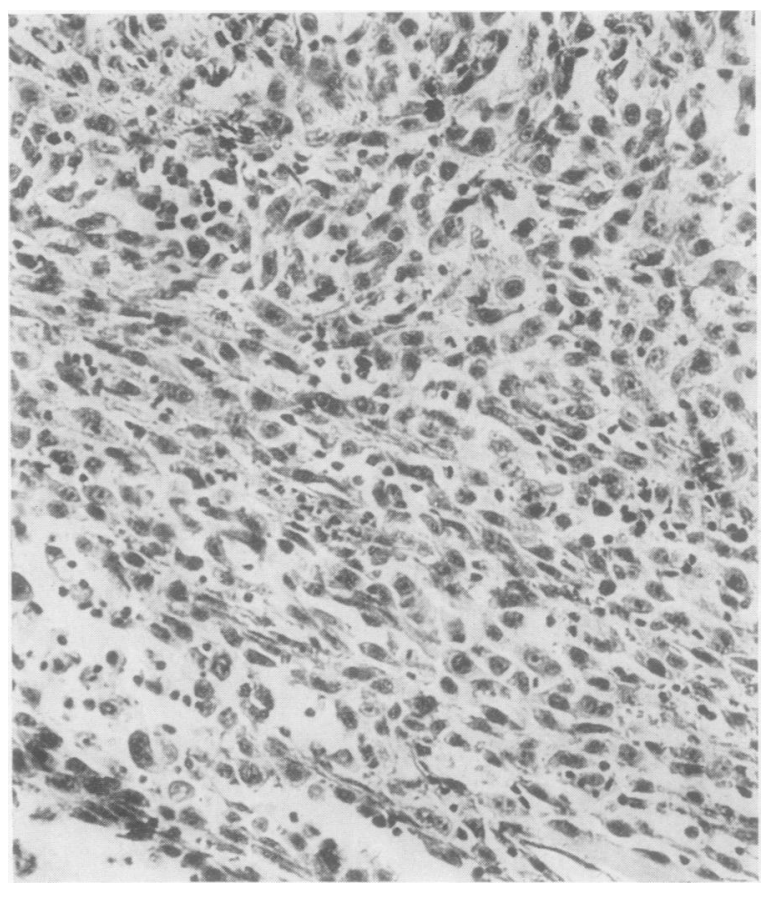

Fig. 15.-Same case as Fig. 14. Squamous carcinoma invading masseter muscle $\times 200$.

alveolar epidermoid carcinomas do spread to the lymph nodes and invade widely.

Everyone with experience of these tumours must have difficulty from time to time in knowing how to use the term "adamantinoma." My own view is that since the histological appearance of the typical example, with columnar cells and stellate reticulum, is so remarkable it is useful to apply the word "adamantinoma" to it; but it must always be remembered that this is not really a very specific label and that it does not necessarily mean that the tumour was derived from the enamel organ. I believe that Case 5 in this communication was an example of a basal cell carcinoma arising in the alveolar mucosa and invading the underlying maxilla in somewhat the same way as the embryonic mucosa invades the developing jaw to form a dental lamina. Basal cell carcinomas arising elsewhere do not show a particular tendency to adopt an adamantinomatous or alveolar pattern when they invade bone, and it would seem that there is something inherent in the oral mucosa which determines the frequency of adoption of that pattern in epithelial tumours of the jaw: it is not, of course, invariable, but it happens very often.

Carcinomas of tooth-germ residues might be expected on general morphological grounds to give 
rise to tumours of columnar, basal cell type or to squamous epithelial variants: it is the variants that occasionally cause histological diagnostic difficulty. In Case 4 it seemed that complete squamous differentiation of an adamantinoma had occurred. It behaved like an adamantinoma and for that reason it seemed to me justifiable to call it an adamantinoma, because every surgeon will know what is meant by that term. For completeness it was only necessary to add that it had undergone squamous metaplasia without any evidence of the development of squamous cell carcinoma.

McWhirter (1950) suggests that rodent ulcers of the skin, adamantinomata, some salivary gland tumours, and bronchial adenoma should all be grouped together as "adnexal tumours," but this only introduces another not very helpful name and has no justification beyond his claim that they react similarly to radiotherapy. I would suggest that we can still use the terms "adamantinoma of the jaw, with characteristic histological pattern," "adamantinoma of the jaw, with squamous metaplasia," "adamantinoma of the maxillary antrum," "adamantinoma of the tibia," "basal cell carcinoma of the skin, with histological adamantinomatous pattern," and so on. There cannot be a single name for any of these things and there is little point in inventing new ones. It is enough to know that all the conditions mentioned above are locally and rather slowly invasive and do not ordinarily metastasize. By contrast, intra-alveolar epidermoid carcinoma invades widely and metastasizes to lymph nodes.
The distinction between the two types is most important but occasionally difficult. One must, as in so many diagnostic problems, consider all the evidence available - clinical, radiological, and histological. Complete reliance on any one of these three means of diagnosis will sooner or later lead to error.

\section{Summary}

The origin and histological structure of epithelial tumours arising in or invading the jaw is discussed.

It is necessary to distinguish between simple epithelial cysts, adamantinomata, and intra-alveolar epidermoid carcinoma because of their differing prognostic significance.

Examples are presented to illustrate the difficulties in histological diagnosis of all three of these groups, and a plea is made for routine histological study in every clinical case of dental cyst, whatever the radiological appearance may be.

I am indebted to Mr. G. M. FitzGibbon, F.R.C.S., for providing me with most of my material and for the use of his records; to Dr. J. W. E. Snawdon for the use of the photograph of Fig. 2; to Dr. C. H. G. Price, Hon. Secretary of the Bristol Bone Tumour Register, for much of the histological material; and to Mr. G. H. Rogers for all the photography. The Bristol Bone Tumour Register is supported by a grant from the British Empire Cancer Campaign Committee.

\section{REFERENCES}

McWhirter, R. (1950). J. Fac. Radiol., Lond., 2, 31.

Toller, P. A. (1948). Proc. roy. Soc. Med., 41, 681.

Willis, R. A. (1948). Pathology of Tumours, p. 635. London. 